\title{
Biological effects of ticagrelor over clopidogrel in patients with stable coronary artery disease and chronic obstructive pulmonary disease
}

\author{
Gianluca Campo 1,2; Francesco Vieceli Dalla Sega ${ }^{2,3}$; Rita Pavasini'; Giorgio Aquila2,3; Francesco Gallo1; Francesca Fortini2,3; \\ Elisabetta Tonet'; Paolo Cimaglia'; Annamaria Del Franco'; Gabriele Pestelli'; Alessandro Pecoraro'; Marco Contoli"; Cristina Balla'; \\ Simone Biscaglia'; Paola Rizzo5; Roberto Ferrari ${ }^{1,2,6}$ \\ ${ }^{1}$ Cardiovascular Institute, Azienda Ospedaliero-Universitaria di Ferrara, Cona (FE), Italy; ${ }^{2}$ Laboratorio per le Tecnologie delle Terapie Avanzate (LTTA) Center, Ferrara, Italy; \\ ${ }^{3}$ Department of Medical Sciences, University of Ferrara, Ferrara, Italy; ${ }^{4}$ Research Centre on Asthma and COPD, Section of Internal and Cardio-Respiratory Medicine, University of \\ Ferrara, Ferrara, Italy; ${ }^{5}$ Department of Morphology, Surgery and Experimental Medicine, University of Ferrara, Ferrara, Italy; ${ }^{6}$ Maria Cecilia Hospital, GVM Care \& Research, E. S.: \\ Health Science Foundation, Cotignola, Italy
}

\begin{abstract}
Summary
Patients with SCAD and concomitant COPD are at high risk of cardiovascular adverse events, due to chronic inflammation, responsible of endothelial dysfunction, oxidative stress and heightened platelet reactivity (PR). The objective of this randomised clinical trial was to test if ticagrelor is superior to clopidogrel in improving endothelial function in patients with stable coronary artery disease (SCAD) and concomitant chronic obstructive pulmonary disease (COPD). Forty-six patients with $S C A D$ and $C O P D$ undergoing percutaneous coronary intervention $(\mathrm{PCl})$ were randomly assigned to receive clopidogrel $(n=23)$ or ticagrelor $(n=23)$ on top of standard therapy with aspirin. The following parameters were assessed at baseline and after 1 month: i) rate of apoptosis and ii) nitric oxide (NO) levels in human umbilical vein endothelial cells (HUVECs), iii) levels of reactive oxygen species (ROS) in peripheral blood mononuclear cell, iv) 29 cytokines/chemokines, v) ontreatment PR. The primary endpoint of the study was the 1-month rate
\end{abstract}

of HUVECs apoptosis. The rate of apoptosis after 1 month was significantly lower in patients treated with ticagrelor $(7.4 \pm 1.3 \%$ vs $9.3 \pm$ $1.5 \%, p<0.001)$, satisfying the pre-specified primary endpoint. In the ticagrelor arm, levels of $\mathrm{NO}$ were higher $(10.1 \pm 2.2 \mathrm{AU}$ vs $8.5 \pm 2.6$ $\mathrm{AU}, \mathrm{p}=0.03)$ while those of $\mathrm{ROS}(4 \pm 1.8 \mathrm{AU}$ vs $5.7 \pm 2.8 \mathrm{AU}, \mathrm{p}=0.02)$ and $P_{2} Y_{12}$ reactivity units $(52 \pm 70 \mathrm{PRU}$ vs $155 \pm 62 \mathrm{PRU}, \mathrm{p}<0.001)$ were lower. There were no differences in cytokines/chemokines levels and aspirin reactivity units between groups. In patients with SCAD and COPD undergoing $\mathrm{PCl}$, ticagrelor, as compared to clopidogrel is superior in improving surrogate markers of endothelial function and on-treatment PR (ClinicalTrials.gov, NCT02519608).

\section{Keywords}

Ticagrelor, clopidogrel, endothelial function, stable coronary artery disease, chronic obstructive pulmonary disease

Financial support:

This study was partially supported by a research grant [ESR-14-10659] from AstraZeneca, which had no role in the collection, analysis, and interpretation of data; writing of the report; and the decision to submit the paper for publication.

Received: December 30, 2016

Accepted after major revision: March 8, 2017

Epub ahead of print: March 23, 2017

https://doi.org/10.1160/TH16-12-0973

Thromb Haemost 2017; 117: $\mathbf{\square}$

Supplementary Material to this article is available online at www.thrombosis-online. com.

\section{Introduction}

Patients with stable coronary artery disease (SCAD) and concomitant chronic obstructive pulmonary disease (COPD) undergoing percutaneous coronary intervention (PCI) show a poor prognosis as compared to those without COPD (1-5). They have higher long-term rates of death, reinfarction and stroke (1-5). It has been suggested that the chronic inflammatory status related to COPD plays a key role, inducing a worsening in arterial stiffness, endothelial dysfunction, platelet reactivity (PR) and atheroma burden $(2,6)$.
Ticagrelor, a newer $\mathrm{P} 2 \mathrm{Y}_{12}$ inhibitor, exerts a more powerful platelet inhibition as compared to clopidogrel $(7,8)$. In addition, recent evidences suggested that ticagrelor may have additional $\mathrm{P} 2 \mathrm{Y}_{12}$-independent biological effects promoting endothelial function homeostasis (7-9). Whether this is present and confirmed also in patients with SCAD and COPD undergoing PCI is unknown.

Thus, the hypothesis of our study was to evaluate whether ticagrelor was superior to clopidogrel in preserving endothelial function and in reducing inflammation and on-treatment PR in this class of patients. 


\section{Material and methods Study design}

The compariso $\mathrm{N}$ between ticAgrelor and clopidogrel effect on endoTHelial, platelet ANd iNflammation parameters in patiEnts with stable coronary artery disease and chronic obstructiVE pulmonaRy disease undergoing percutaneous coronary intervention (NATHAN-NEVER) is an investigator-initiated, prospective, single-centre, randomised, open-label phase II trial involving consecutive SCAD patients with concomitant COPD referred to our Cardiovascular Centre between September 2015 and June 2016 ( Figure 1). The protocol has been approved by the corresponding Ethics Authority (Comitato Etico Unico della Provincia di Ferrara). All patients gave their written informed consent. The study is registered at www.clinicaltrials.gov with the identifier NCT02519608.

\section{Study population}

Inclusion criteria were: i) age $>18$ years; ii) ability to provide informed written consent; iii) SCAD diagnosis requiring coronary artery angiography (CAA) and PCI; iv) COPD diagnosis confirmed by spirometry (stable phase in the last 3 months). SCAD diagnosis and clinical indication to CAA was based according to current guidelines criteria (1). Briefly, we included patients with stable angina pectoris requiring CAA because symptoms were present despite optimal medical treatment or because of the presence of high risk of event after risk stratification (1). The diagnosis of COPD was based on spirometry data according to international guidelines and in particular by the presence of not fully reversible airflow limitation defined as a ratio between forced expiratory volume at 1 second (FEV1) and forced vital capacity (FVC) ratio $<0.7$ after the administration of a bronchodilator (3). An independent blinded reviewer (MC) analysed spirometry and documentation available to confirm COPD diagnosis and to adjudicate severity of airflow limitation based on post-bronchodilator FEV1 (from mild to very severe) (3). Main exclusion criteria were: i) prior administration of P2Y12 inhibitor (clopidogrel, ticlopidine, prasugrel, ticagrelor) or of anticoagulant drugs (e.g. warfarin); ii) known intolerance to clopidogrel or ticagrelor; iii) prior intracranial haemorrhage; iv) cerebrovascular accident and/or active major bleeding and/or major surgery within the last 30 days and v) other known inflammatory chronic disorders. A fully detailed list of inclusion and exclusion criteria is reported in the Suppl. Material (available online at www.thrombosis-online.com).

\section{Randomisation and interventions}

Forty-six patients were enrolled and randomly assigned to receive, on top of aspirin, clopidogrel or ticagrelor. The P2Y12 inhibitors were administered at the doses currently approved for the daily clinical practice (clopidogrel: $600 \mathrm{mg}$ loading dose $+75 \mathrm{mg}$ daily; ticagrelor: $180 \mathrm{mg}$ loading dose $+90 \mathrm{mg}$ twice daily). The dual antiplatelet treatment was recommended for at least six months, preferably 12 months. Randomisation was performed by an inde- pendent study coordinator via sealed envelopes. To minimise potential confounding effect, randomisation was stratified according to the presence of diabetes (yes vs no) and COPD severity (mild vs moderate vs severe vs very severe). Treatment adherence was assessed by the self-report of the number of missed doses of the study drug per week. PCI procedure and stent implantation (second generation drug-eluting stent) were performed according to standard techniques and institutional protocols (10-12). The remaining cardiovascular treatment was based according to current guidelines; drugs were titrated before PCI and any change between PCI and 1-month visit was strongly discouraged.

\section{Blood samples}

Venous blood samples were collected at baseline (before PCI and study drug administration) and at the 1-month visit from an antecubital vein using a 21-gauge needle. The first $2-4 \mathrm{ml}$ of blood was discarded. All patients underwent blood sampling in the early morning (8.00 AM - 10.00 AM), after at least 30 minutes of rest and 2 hours (h) of fasting. Of note, the 1-month blood sample was collected $12 \mathrm{~h}$ after the last maintenance dose of ticagrelor and 24 $\mathrm{h}$ after the last dose of clopidogrel.

\section{Endothelial function analysis}

Endothelial function was evaluated with three different parameters. Firstly, we assessed the rate of apoptosis in human umbilical vein endothelial cells (HUVECs) after 48-hour incubation with $20 \%$ serum from patients $(13,14)$. The rate of apoptosis was determined by flow cytometry, defining the number of annexin $\mathrm{V}$ and propidium iodide positive cells $(13,14)$. Apoptosis rate was expressed as percentage (\%) of positive cells as compared to the total number of cells $(13,14)$. Secondly, we evaluated nitric oxide (NO) levels in HUVECs treated with patient's serum (15). Finally, we determined the intracellular levels of reactive oxygen species (ROS) in peripheral blood mononuclear cell (PBMC) isolated from patients (16). Both NO and ROS levels were expressed as arbitrary units (AU). > Figure 2 shows a graphic representation of all the tests performed in two representative patients. The detailed description of the assays is available in the Suppl. Material (available online at www.thrombosis-online.com).

\section{Inflammation parameters}

Twenty-nine human cytokines/chemokines levels were determined in all patients with a bead-based multiplex immunoassay. A detailed description of the assays is reported in the Suppl. Material (available online at www.thrombosis-online.com).

\section{Platelet function analysis}

On-treatment PR was assessed by the VerifyNow ${ }^{\mathrm{mm}}$ system (Accumetrics, San Diego, CA, USA), using specific assays to test aspirin (VerifyNow Aspirin $^{\mathrm{nm}}$ ) and $\mathrm{P} 2 \mathrm{Y}_{12}$ inhibitors (VerifyNow P2Y12 ${ }^{\mathrm{mm}}$ ) $(17,18)$. The results were expressed as aspirin reaction unit (ARU) 


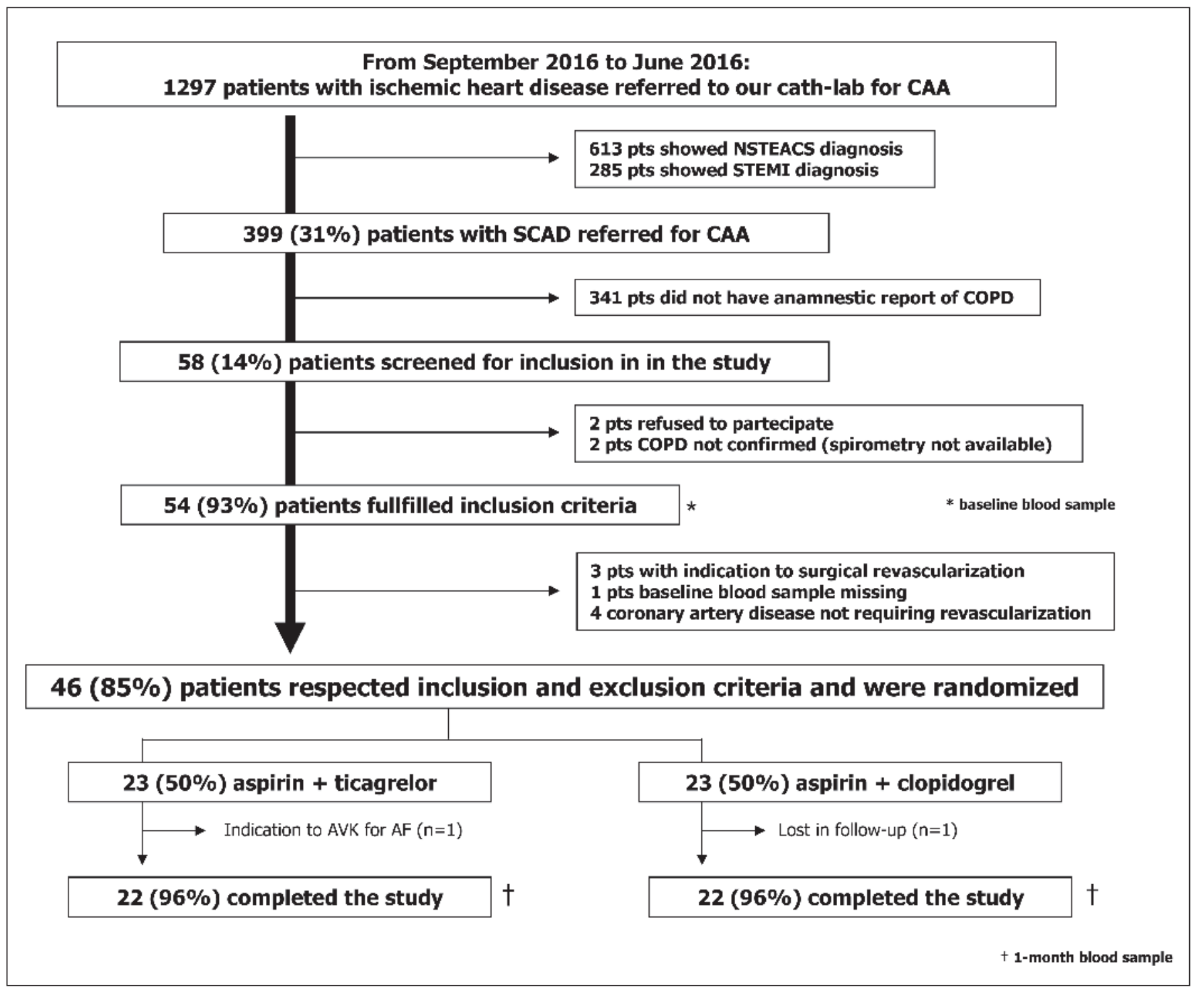

Figure 1: Study flow diagram. CAA: coronary artery angiography. NSTEACS: not ST-segment elevation acute coronary syndrome. STEMI: ST-segment elevation myocardial infarction. SCAD: stable coronary artery disease. COPD: chronic obstructive pulmonary disease. AVK: antagonist vitamin K. AF: atrial fibrillation.

and $\mathrm{P}_{2} \mathrm{Y}_{12}$ reaction units (PRU), respectively $(17,18)$. As previously established (19), we defined high on-treatment PR in presence of 1-month PRU value $>208$.

\section{Endpoints of the study}

The primary endpoint of the study was 1-month rate of apoptosis in HUVECs. Secondary endpoints included 1-month levels of: i) NO in HUVECs; ii) intracellular ROS in PBMC; iii) cytokines/ chemokines in serum; iv) PRU and ARU. Safety endpoints were the 1-month occurrence of ischaemic and bleeding adverse events (according to the Academic Research Consortium and Bleeding Academic Research Consortium classification) $(20,21)$.

\section{Statistical analysis}

According to previous studies $(13,14)$, we hypothesised a baseline rate of apoptosis in HUVECs of 10-11\% with a standard deviation (SD) of $2.5 \%$. We arbitrarily assume that ticagrelor would decrease the rate of apoptosis by $20 \%$ vs clopidogrel, therefore at least 20 patients per group were required for $80 \%$ power and a two-sided $\alpha$-value of 0.05 . Continuous variables with normal distribution were expressed as means $\pm \mathrm{SD}$. Continuous variables with a nonnormal distribution were expressed as median and interquartile range. Normal distribution of the variables was tested with the Kolmogorov-Smirnov test. The variables normally distributed were compared by t-test and one-way ANOVA; otherwise the Mann-Whitney U and Kruskal-Wallis tests were used. Categorical 


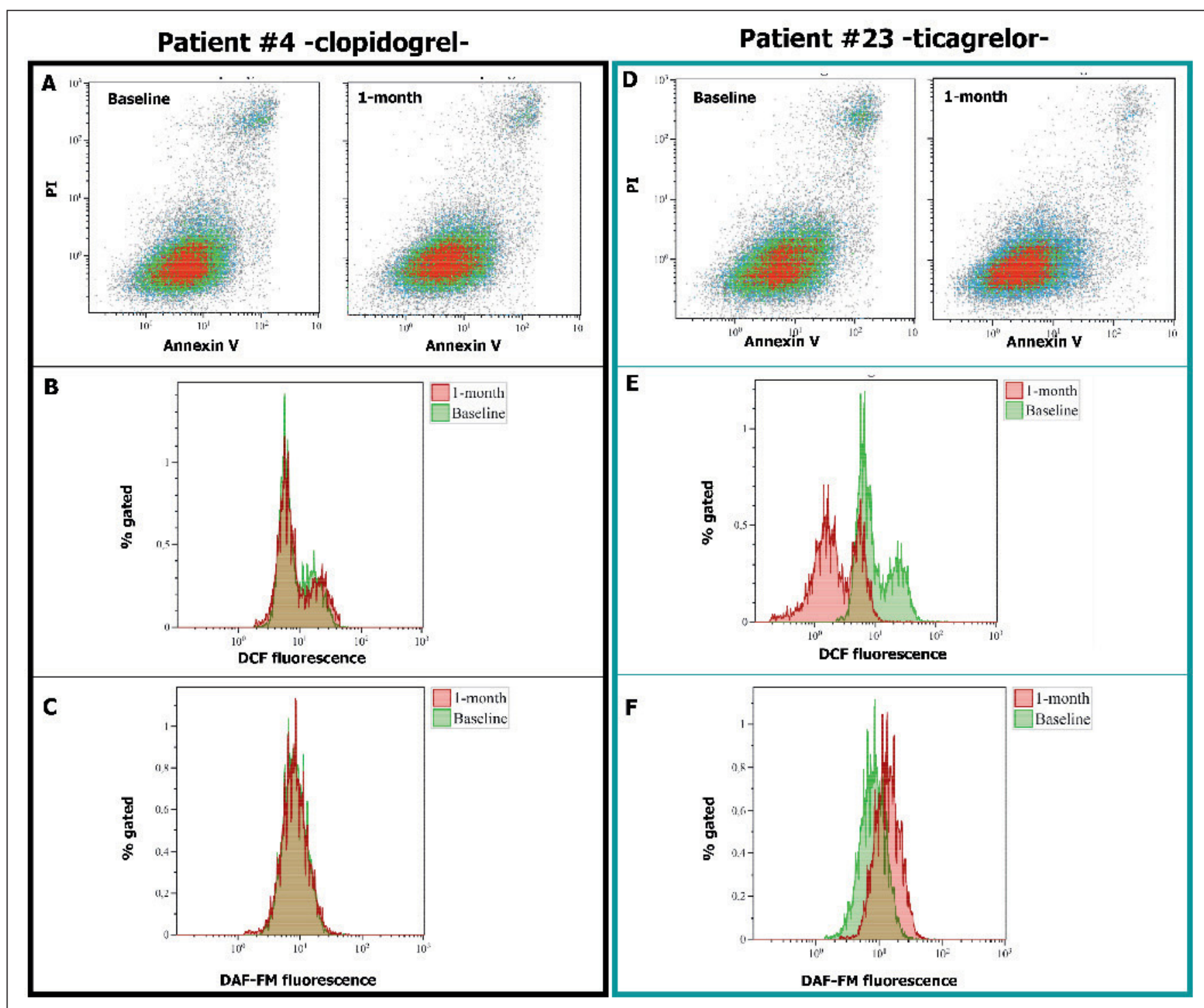

Figure 2: Schematic representation of endothelial function assays in two patients. We reported the endothelial function assays of patient \#4 (randomised to clopidogrel) and of patient \#23 (randomised to ticagrelor) both at baseline and after 1 month. A and D) Rate of apoptosis. B and E) ROS generation. $\mathrm{C}$ and F) NO levels. Of note, in Figures $\mathrm{B}, \mathrm{C}, \mathrm{E}$ and $\mathrm{F}$ baseline sample is pink, 1-month sample is green and the overlap zone is brown. PI: propidium iodide. DCF: $2^{\prime}, 7^{\prime}$ 'dichlorofluorescin diacetate. DAF-FM: 4-amino5-methylamino-2',7' difluorofluorescein diacetate. variables were summarised in terms of numbers and percentages and were compared by using the two-sided Fisher's exact test. Correlation of continuous data was performed using Pearson or Spearman correlation, as appropriate. For all comparisons, a p-value of $<0.05$ was considered statistically significant. When appropriate, $95 \%$ confidence intervals (CIs) were calculated. All analyses were performed with STATISTICA 8 (Statsoft Inc, Tulsa, OK, USA).

\section{Results}

Table 1 shows baseline characteristics of the enrolled patients. They were all Caucasian with mean age of $68 \pm 9$ years. Fifteen
(32\%) of the 46 patients were diabetic. Smoking history was present in all patients, $11(24 \%)$ were current smokers. PCI procedure and stent implantation was successful in all patients. As showed in - Figure 1, 23 (50\%) patients were assigned to clopidogrel, whereas $23(50 \%)$ to ticagrelor. Cardiovascular risk factors, severity of airways obstruction, cardiovascular and respiratory therapy did not significantly differ between groups.

\section{Primary outcome}

At baseline, rate of apoptosis was $10.2 \pm 2 \%$ and it did not differ between clopidogrel vs ticagrelor group ( $\mathrm{p}=0.6)(\triangleright$ Table 2). On the contrary, after 1 month of treatment the rate of apoptosis in the 
Table 1: Characteristics of the study population.

\begin{tabular}{|c|c|c|c|}
\hline & $\begin{array}{l}\text { Clopidogrel } \\
(n=23)\end{array}$ & $\begin{array}{l}\text { Ticagrelor } \\
(n=23)\end{array}$ & P-value \\
\hline Age, (years) & $67 \pm 8$ & $69 \pm 9$ & 0.4 \\
\hline Male sex, no. (\%) & $19(83)$ & $20(87)$ & 0.5 \\
\hline $\mathrm{BMI},\left(\mathrm{Kg} / \mathrm{m}^{2}\right)$ & $28 \pm 5$ & $28 \pm 3$ & 0.8 \\
\hline Diabetes, no. (\%) & $7(30)$ & $8(35)$ & 0.6 \\
\hline Hypertension, no. (\%) & $20(87)$ & $18(78)$ & 0.3 \\
\hline Hyperlipidaemia, no. (\%) & $17(74)$ & $15(65)$ & 0.4 \\
\hline Current smoker, no. (\%) & $6(26)$ & $5(22)$ & 0.4 \\
\hline from years & $43 \pm 10$ & $42 \pm 11$ & 0.6 \\
\hline Previous smoker, no. (\%) & $17(74)$ & $18(78)$ & 0.4 \\
\hline stopped from years & $10 \pm 8$ & $11 \pm 9$ & 0.7 \\
\hline smoked for years & $31 \pm 10$ & $32 \pm 13$ & 0.8 \\
\hline pack/years & $42[26-50]$ & $40[20-58]$ & 0.3 \\
\hline Prior MI, no. (\%) & $5(22)$ & $4(17)$ & 0.8 \\
\hline Prior PCl, no. (\%) & $6(26)$ & $6(26)$ & 0.9 \\
\hline Prior CABG, no. (\%) & $0(0)$ & $0(0)$ & 0.9 \\
\hline \multicolumn{4}{|l|}{ Laboratory data at baseline } \\
\hline White blood cells, $(\mathrm{U} / \mu \mathrm{l})$ & $8 \pm 1$ & $8 \pm 2$ & 0.4 \\
\hline Haemoglobin, (g/dl) & $13 \pm 1$ & $13 \pm 1$ & 0.6 \\
\hline Platelets $(\mathrm{U} / \mu \mathrm{l})$ & $207 \pm 64$ & $204 \pm 60$ & 0.9 \\
\hline Creatinine clearance, (ml/min) & $75 \pm 8$ & $70 \pm 10$ & 0.3 \\
\hline $\operatorname{LVEF}(\%)$ & $53 \pm 8$ & $54 \pm 9$ & 0.5 \\
\hline \multicolumn{4}{|l|}{ COPD } \\
\hline Years from diagnosis, (years) & $11 \pm 5$ & $11 \pm 6$ & 0.9 \\
\hline Mild AO no. (\%) & $10(43)$ & $11(48)$ & 0.9 \\
\hline Moderate AO no. (\%) & $12(52)$ & $11(48)$ & 0.9 \\
\hline Severe AO no. (\%) & $1(4)$ & $1(4)$ & 0.9 \\
\hline Very severe $\mathrm{AO}$ no. $(\%)$ & $0(0)$ & $0(0)$ & 0.9 \\
\hline \multicolumn{4}{|l|}{ Cardiovascular therapy, no. (\%) } \\
\hline Aspirin & $23(100)$ & $23(100)$ & 0.9 \\
\hline Beta-blocker & $17(74)$ & $17(74)$ & 0.9 \\
\hline ACE inhibitor/ARB & $21(91)$ & $20(87)$ & 0.9 \\
\hline Statin & $23(100)$ & $23(100)$ & 0.9 \\
\hline Calcium channel blockers & $8(35)$ & $9(39)$ & 0.7 \\
\hline Nitrates & $3(13)$ & $3(13)$ & 0.9 \\
\hline Proton pump inhibitor * & $8(35)$ & $9(39)$ & 0.7 \\
\hline \multicolumn{4}{|l|}{ Respiratory therapy, no. (\%) } \\
\hline Inhaled corticosteroids & $4(17)$ & $5(22)$ & 0.9 \\
\hline Short-acting beta2 agonist & $8(35)$ & $7(30)$ & 0.9 \\
\hline Long-acting beta2 agonist & $6(26)$ & $8(35)$ & 0.9 \\
\hline Long-acting muscarinic antagonist & $9(39)$ & $8(35)$ & 0.9 \\
\hline \multicolumn{4}{|c|}{$\begin{array}{l}\text { BMI: body mass index. MI: myocardial infarction. PCI: percutaneous coronary intervention. CABG: coronary } \\
\text { artery bypass graft. LVEF: left ventricle ejection fraction. COPD: chronic obstructive pulmonary disease. } \\
\text { AO: airways obstruction. ACE: angiotensin converting enzyme. ARB: angiotensin II receptor blocker. *: } \\
\text { pantoprazole was the only proton pump inhibitor administered. }\end{array}$} \\
\hline
\end{tabular}




\begin{tabular}{|c|c|c|c|c|c|}
\hline & $\begin{array}{l}\text { Clopidogrel } \\
(n=23)\end{array}$ & $\begin{array}{l}\text { Ticagrelor } \\
(n=23)\end{array}$ & p1 & p2 & p3 \\
\hline \multicolumn{6}{|l|}{ Primary endpoint } \\
\hline Baseline apoptosis rate, (\%) & $10.3 \pm 2$ & $10.1 \pm 2$ & 0.6 & 0.09 & 0.0001 \\
\hline 1-month apoptosis rate, (\%) & $9.3 \pm 1.5$ & $7.4 \pm 1.3$ & $<0.001$ & & \\
\hline \multicolumn{6}{|l|}{ Secondary endpoints } \\
\hline Baseline NO, (AU) & $8.5 \pm 1.4$ & $8.7 \pm 1.8$ & 0.7 & 0.8 & 0.03 \\
\hline 1-month NO, (AU) & $8.5 \pm 2.6$ & $10.1 \pm 2.2$ & 0.03 & & \\
\hline Baseline ROS, (AU) & $6.2 \pm 3.4$ & $6.1 \pm 2.5$ & 0.9 & 0.5 & 0.002 \\
\hline 1-month ROS, (AU) & $5.7 \pm 2.8$ & $4 \pm 1.8$ & 0.02 & & \\
\hline Baseline aspirin reactivity units, (ARU) & $447 \pm 58$ & $450 \pm 52$ & 0.8 & 0.3 & 0.01 \\
\hline 1-month aspirin reactivity units, (ARU) & $426 \pm 69$ & $402 \pm 75$ & 0.5 & & \\
\hline Baseline P2Y12 reactivity units, (PRU) & $235 \pm 38$ & $240 \pm 45$ & 0.6 & $<0.001$ & $<0.001$ \\
\hline 1-month P2Y12 reactivity units, (PRU) & $155 \pm 62$ & $52 \pm 70$ & $<0.001$ & & \\
\hline \multicolumn{6}{|c|}{$\begin{array}{l}\text { p1: for the comparison clopidogrel vs ticagrelor. p2: for the comparison clopidogrel baseline vs clopidogrel } \\
\text { 1-month. p3: for the comparison ticagrelor baseline vs ticagrelor 1-month. NO: nitric oxide. ROS: reac- } \\
\text { tive oxygen species. AU: arbitrary units. }\end{array}$} \\
\hline
\end{tabular}

Table 2: Principal endpoints of the study.

Table 2: Principal endpoints of the study.

ticagrelor group was significantly lower compared with the clopidogrel group $(7.4 \pm 1.3 \%$ vs $9.3 \pm 1.5 \%, \mathrm{p}<0.001$, respectively), satisfying our pre-specified primary endpoint ( $>$ Table 2, $>$ Figure 3).

\section{Secondary outcomes}

\section{Nitric oxide}

Baseline NO levels did not differ between ticagrelor and clopidogrel group ( $>$ Table 2 ). In the ticagrelor arm, but not in the clopi-

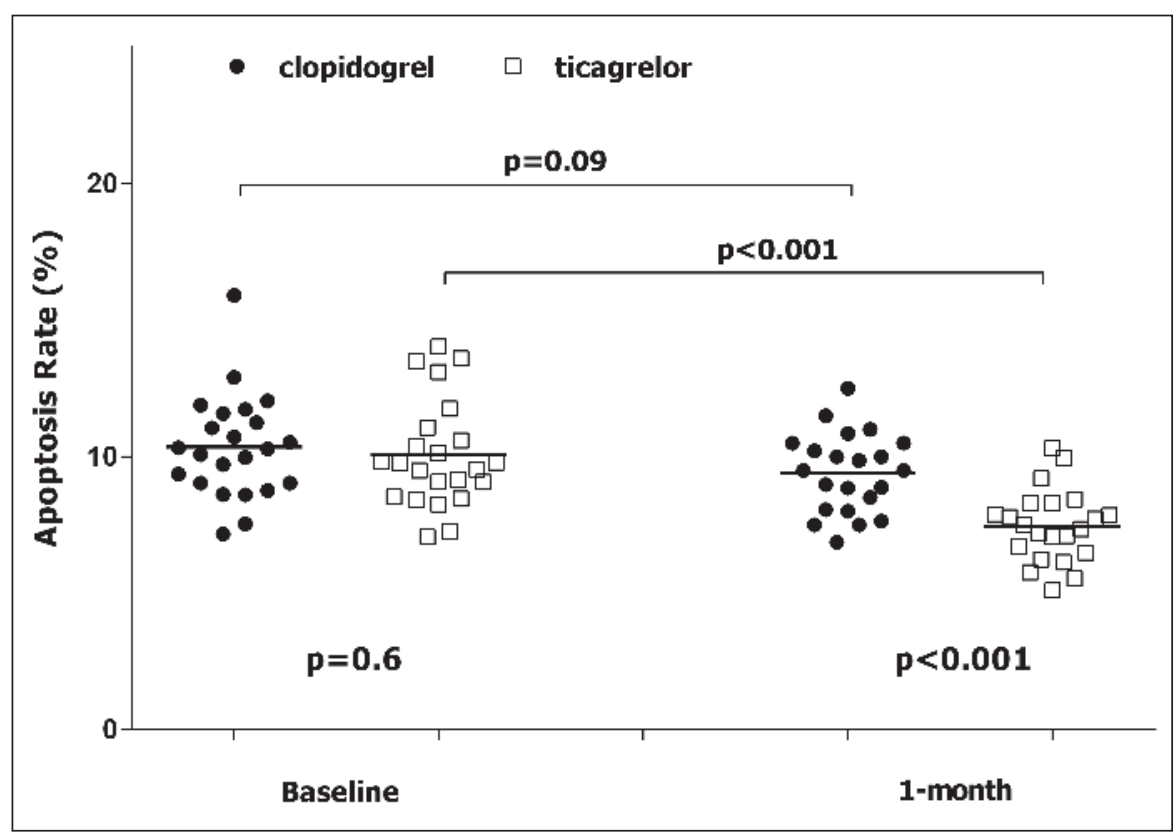

Figure 3: Apoptosis rate. Apoptosis is expressed as percentage of Annexin $V$ positive cells on the total number of cells. Black circle: patient randomised to clopidogrel. White square: patient randomised to ticagrelor. 
Figure 4: $P 2 Y_{12}$ reaction units. Black circle: patient randomised to clopidogrel. White square: patient randomised to ticagrelor.

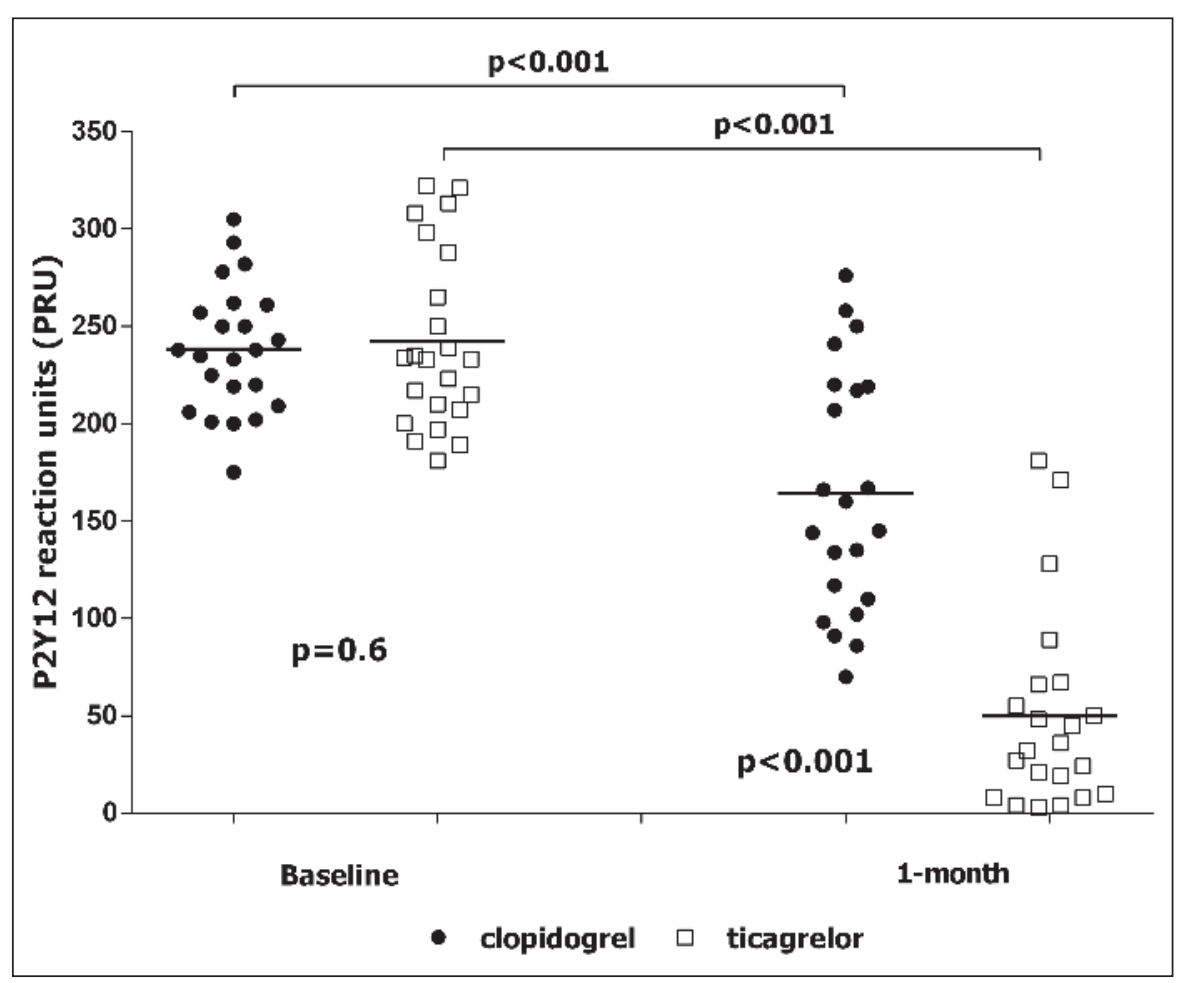

dogrel arm, NO levels significantly increased from baseline to 1 month ( $\mathrm{p}=0.03)$ ( Table 2). Consecutively, 1-month NO levels in the ticagrelor arm were significantly higher as compared to those of the clopidogrel arm $(10.1 \pm 2.2 \mathrm{AU}$ vs $8.5 \pm 2.6 \mathrm{AU}, \mathrm{p}=0.03)$ ( Table 2). At 1 month, the rate of apoptosis and NO levels were inversely related $\left(r=-0.52, r^{2}=0.27, p=0.0003\right)$. Similar findings were observed considering the change from baseline to 1 month of the rate of apoptosis and of NO levels $\left(r=-0.53, r^{2}=0.29, p=0.0002\right)$. After stratification in tertiles of 1-month rate of apoptosis, we observed a significant decrease in $\mathrm{NO}$ values from the lowest to the highest tertiles ( $\mathrm{p}$ for trend 0.004, Suppl. Figure 1, available online at www.thrombosis-online.com).

\section{Radical oxygen species}

As for NO, ROS levels did not differ between groups at baseline, but after 1 month ROS levels significantly decreased only in the ticagrelor group ( $\mathrm{p}=0.02)$ ( $\$$ Table 2). 1-month ROS levels were lower in the ticagrelor arm as compared to clopidogrel one ( $4 \pm 1.8$ $\mathrm{AU}$ vs $5.7 \pm 2.8 \mathrm{AU}, \mathrm{p}=0.02$ ) ( Table 2). At 1 month, the rate of apoptosis and ROS levels were directly related $\left(r=0.34, r^{2}=0.12\right.$, $\mathrm{p}=0.02$ ). The change from baseline to 1 month of rate of apoptosis and ROS levels confirmed the relationship $\left(\mathrm{r}=0.51, \mathrm{r}^{2}=0.26\right.$, $\mathrm{p}=0.0004)$. ROS levels increased significantly across tertiles of 1 -month rate of apoptosis (p for trend 0.009, Suppl. Figure 2, available online at www.thrombosis-online.com).

\section{Cytokines/chemokines}

Serum levels of cytokines/chemokines did not differ between groups at baseline and after 1 month. The 1-month treatment with ticagrelor or clopidogrel did not influence their values. The details of the 29 cytokines/chemokines measured are reported in Suppl. Table 1 (available online at www.thrombosis-online.com).

\section{Aspirin and $\mathrm{P}_{2} \mathrm{Y}_{12}$ reactivity unit}

As expected, baseline values of ARU and PRU did not differ between groups ( Table 2). Randomisation to clopidogrel or ticagrelor did not influence 1-month ARU values ( $447 \pm 58$ ARU vs $450 \pm 52$ ARU, $\mathrm{p}=0.5$, respectively). On the contrary, after 1 month, values of PRU were significantly lower in the ticagrelor group as compared to the clopidogrel arm $(57 \pm 70$ PRU vs $148 \pm$ 62 PRU, p $<0.001$, respectively) ( Table 2, Figure 4). Overall, we identified eight (17\%) patients with high on-treatment PR. They were all in the arm randomised to clopidogrel $(35 \%$ vs $0 \%$, $\mathrm{p}=0.01$ ). Suppl. Table 2 (available online at www.thrombosis-on line.com ) reported the correlation between PRU, rate of apoptosis, $\mathrm{NO}$ and ROS values. We did not find any significant correlation neither at baseline nor at 1 month (see Suppl. Table 2, available online at www.thrombosis-online.com).

\section{Safety and tolerability}

No serious adverse events were reported. One patient of the clopidogrel arm refused to repeat the blood sample at the 1-month visit. 
Due to the occurrence of atrial fibrillation and to the need of anticoagulant therapy, one patient of the ticagrelor arm discontinued the study treatment ( Figure 1). Finally, shortness of breath was reported in two (8\%) out of 23 patients treated with ticagrelor, without the need to drug discontinuation.

\section{Discussion}

The main finding of our study is that 1-month treatment with ticagrelor, compared with clopidogrel, significantly improves endothelial function and on-treatment PR of patients with SCAD and COPD undergoing successful PCI and stent implantation.

The strengths and novelties of this report are the translational approach of the hypothesis and the particular features of the study population.

Indeed, COPD is a high-risk comorbidity for patients with SCAD. From a clinical point of view, COPD is associated with a higher risk of recurrent ischaemic events and of all-cause mortality, independently from clinical presentation, interventional treatment, cardiovascular pharmacotherapy, COPD diagnosis (clinical vs spirometry) and respiratory treatment (2, 5, 22-24). From a pathophysiological point of view, COPD is associated with a chronic inflammation, endothelial dysfunction and persistently higher oxidative stress and platelet reactivity $(2,6)$. Thus, we may speculate that patients with concomitant SCAD and COPD have both a "clinical" and "biological" profile that may benefit from ticagrelor administration.

\section{What is known about this topic?}

- Patients with stable coronary artery disease (SCAD) and concomitant chronic obstructive pulmonary disease (COPD) are at higher adverse cardiovascular events as compared to those without COPD.

- The negative prognostic effect is due to chronic inflammation status associated with COPD, responsible of endothelial dysfunction, oxidative stress and heightened platelet reactivity.

- Preliminary evidences suggested that ticagrelor may have additional $\mathrm{P}_{2} \mathrm{Y}_{12}$-independent biological effects promoting endothelial function homeostasis

\section{What does this paper add?}

- This is the first study assessing the potential ticagrelor benefit in patients at higher risk not for the cardiac condition (SCAD vs acute coronary syndrome), but for the comorbidity (COPD).

- This is the first randomised clinical trial showing that ticagrelor exerts a higher improvement of the endothelial function as well as of the on-treatment platelet reactivity as compared to clopidogrel.

- Future studies will test if the observed biological benefit could be translated in an advantage for the reduction of the risk of clinical events in patients with SCAD and concomitant COPD undergoing $\mathrm{PCl}$.
Ticagrelor, when compared to clopidogrel, significantly reduced the primary efficacy endpoint of death from vascular causes, myocardial infarction and stroke in patients with acute coronary syndrome (ACS) (25). Surprisingly, ticagrelor also reduced cardiovascular mortality, an effect never seen with the other $\mathrm{P} \mathrm{Y}_{12}$ inhibitors, suggesting an additional benefit independently by $\mathrm{P}_{2} \mathrm{Y}_{12}$ inhibition $(9,26)$. Ticagrelor inhibits adenosine cell uptake (Suppl. Figure 3, available online at www.thrombosis-online. com) $(7,9,26)$ and it induces adenosine triphosphate (ATP) release from human red blood cells (Suppl. Figure 3, available online at www.thrombosis-online.com) (7). The result is circulating plasma levels of adenosine high enough to exert several biological effects including vasodilation by nitric oxide -dependent and -independent pathways and inhibition of neutrophil trafficking, granule release, production of ROS and inflammatory mediators (Suppl. Figure 3, available online at www.thrombosis-online.com) (7). Furthermore, ticagrelor inhibits the adenosine diphosphate (ADP)-induced vasoconstriction mediated by $\mathrm{P}_{2} \mathrm{Y}_{12}$ receptors in vascular smooth muscle cells (Suppl. Figure 3, available online at www.thrombosis-online.com) (7).

These clinical and biological findings were the background for the NATHAN NEVER study.

We focus our attention on a peculiar study population, where ticagrelor $\mathrm{P}_{2} \mathrm{Y}_{12}$-independent effects may be amplified and widely beneficial. The ability by antiplatelet agents to improve endothelial function is known $(9,27)$. Recently, Mangiacapra et al. showed that both ticagrelor and clopidogrel (150 mg maintenance dose) were able to modulate endothelial function in patients with SCAD and type 2 diabetes mellitus (28). Diabetic patients were selected because they are a well established subgroup of patients with heightened platelet reactivity and endothelial dysfunction, independently by cardiologic clinical presentation (28). In this setting, they found that ticagrelor is able to provide, besides a more profound platelet inhibition, a significant improvement in endothelial function and endothelium-independent brachial artery reactivity (28). Our study confirms and expands these findings. We showed that ticagrelor reduces endothelial apoptosis in patients with concomitant COPD and SCAD. The improvement in endothelial dysfunction was higher with ticagrelor as compared to clopidogrel and not strictly related to the extent of platelet inhibition. It is important to note that it was associated with concomitant improvement of NO generation and attenuation of ROS production, suggesting a preservation of endothelial function. These findings are important, because the reduction of the rate of endothelial apoptosis with either angiotensin converting enzyme inhibitor or statin has been linked to a slowdown in the progression of atherosclerosis and, eventually, of cardiovascular events $(29,30)$.

Our protocol does not allow having information on the intimate mechanism of ticagrelor antiapoptotic effect. This effect could be related to adenosine, but we may not exclude the presence of other mechanisms not already identified. Of note, this seems to be mediated through mechanisms beyond the platelet inhibition (1-month PRU values did non correlate with the rate of apoptosis, NO and ROS generation) and other than inhibition of inflammation. We measured 29 different cytokines and chemo- 
kines and after 1 month of treatment their values were unmodified. A previous study in ACS patients who received 4 weeks of treatment with aspirin plus either ticagrelor (90 mg twice daily or $180 \mathrm{mg}$ twice daily) or clopidogrel (75 mg once daily) also failed to show an effect on inflammation (31). We are well aware that our study population is small and that the short-term treatment period may have been insufficient for optimal assessment of differences in inflammatory biomarkers. Nevertheless, it is similarly plausible that the difference in efficacy between ticagrelor and clopidogrel may be minimal or absent and that ticagrelor $\mathrm{P} 2 \mathrm{Y}_{12}$-independent effects may be mediated through mechanisms other than inhibition of inflammation.

As expected, ticagrelor induced a stronger inhibition of $P R$ than clopidogrel. This effect is also relevant, being those with COPD a subgroup of patients with heightened PR (32). In our study population, all patients showing sub-optimal platelet inhibition were randomised to clopidogrel, whereas in the ticagrelor arm all patients showed an optimal platelet inhibition. This finding deserves special attention because high on-treatment PR remains an independent predictor of ischaemic adverse events, including cardiac death and myocardial infarction (33).

\section{Study limitations}

A major limitation of this study is the lack of adenosine determination in patient's serum. For this reason, we are not able to directly associate our findings to the ticagrelor-induced increase in adenosine levels. Secondly, we are well aware that endothelial function could be assessed with different techniques (e.g. flow-mediated dilation). We decided to use the rate of apoptosis in HUVECs because this methodology has been validated in previous studies with reliable results and it is related to clinical outcome (13, 14). Thirdly, we decided to design a trial including only COPD patients with a randomisation 1: 1 . Of note, a cross-over design or the enrolment of SCAD patients without COPD would be interesting to corroborate our findings. Nevertheless, this is only a small "proof-of-concept" hypothesis-generating study. We demonstrated a "biological" benefit from ticagrelor administration in patients with concomitant SCAD and COPD. If this "biological" effect will be translated in a "clinical" benefit is unknown and proper clinical trials in this population are needed. Similarly, we included SCAD patients and our findings could not be transferred to ACS patients with concomitant COPD.

\section{Conclusions}

Our study showed that, in patients with concomitant SCAD and COPD undergoing PCI, 1-month treatment with ticagrelor, as compared to clopidogrel, is associated with an improvement in the values of surrogate markers of endothelial function, besides a higher platelet inhibition.

\section{Conflicts of interest}

Gianluca Campo has received honoraria for lectures from Astrazeneca, Menarini, Abbott Vascular, Boston Scientific. Roberto Fer- rari has received honoraria for lectures from Servier. The other Authors did not report disclosures.

\section{References}

1. 2014 ESC/EACTS Guidelines on myocardial revascularisation: The Task Force on Myocardial Revascularisation of the European Society of Cardiology (ESC) and the European Association for Cardio-Thoracic Surgery (EACTS)Developed with the special contribution of the European Association of Percutaneous Cardiovascular Interventions (EAPCI). Eur Heart J 2014; 35: 2541-2619.

2. Campo G, Pavasini R, Malagù $M$, et al. Chronic obstructive pulmonary disease and ischemic heart disease comorbidity: overview of mechanisms and clinical management. Cardiovasc Drugs Ther 2015; 29: 147-157.

3. From the Global Strategy for the Diagnosis, Management and Prevention of COPD, Global Initiative for Chronic Obstructive Lung Disease (GOLD), updated 2014.

4. Celli BR, Decramer M, Wedzicha JA, et al. An Official American Thoracic Society/European Respiratory Society Statement: Research questions in chronic obstructive pulmonary disease. Am J Respir Crit Care Med 2015; 191: e4-e27.

5. Campo G, Guastaroba P, Marzocchi A, et al. Impact of COPD on long-term outcome after ST-segment elevation myocardial infarction receiving primary percutaneous coronary intervention. Chest 2013; 144: 750-757.

6. Fabbri LM, Rabe KF. From COPD to chronic systemic inflammatory syndrome? Lancet 2007; 370: 797-799.

7. Cattaneo M, Schulz R, Nylander S. Adenosine-mediated effects of ticagrelor: evidence and potential clinical relevance. J Am Coll Cardiol 2014; 63: 2503-2509.

8. Schnorbus B, Daiber A, Jurk K, et al. Effects of clopidogrel, prasugrel and ticagrelor on endothelial function, inflammatory and oxidative stress parameters and platelet function in patients undergoing coronary artery stenting for an acute coronary syndrome. A randomised, prospective, controlled study. BMJ Open 2014; 4: e005268.

9. Fromonot J, Dignat-Georges F, et al. Ticagrelor Improves Peripheral Arterial Function in Acute Coronary Syndrome Patients: Relationship With Adenosine Plasma Level. J Am Coll Cardiol 2016; 67: 1967-1968.

10. Campo G, Pavasini R, Barbetta C, et al. Predischarge screening for chronic obstructive pulmonary disease in patients with acute coronary syndrome and smoking history. Int J Cardiol 2016; 222: 806-812.

11. Campo G, Morciano G, Pavasini R, et al. Fo ATP synthase C subunit serum levels in patients with ST-segment Elevation Myocardial Infarction: Preliminary findings. Int J Cardiol 2016; 221: 993-997.

12. Biscaglia S, Campo G, Pavasini R, et al. Occurrence, causes, and outcome after switching from ticagrelor to clopidogrel in a real-life scenario: data from a prospective registry. Platelets 2016; 27: 484-487.

13. Valgimigli M, Agnoletti L, Curello S, et al. Serum from patients with acute coronary syndromes displays a proapoptotic effect on human endothelial cells: a possible link to pan-coronary syndromes. Circulation 2003; 107: 264-270.

14. Agnoletti L, Curello S, Bachetti T, et al. Serum from patients with severe heart failure downregulates eNOS and is proapoptotic: role of tumor necrosis factoralpha. Circulation 1999; 100: 1983-1991.

15. Nakatsubo N, Kojima H, Kikuchi K, et al. Direct evidence of nitric oxide production from bovine aortic endothelial cells using new fluorescence indicators: diaminofluoresceins. FEBS Lett 1998; 427: 263-266.

16. Sarkar M, Varshney R, Chopra M, et al. Flow-cytometric analysis of reactive oxygen species in peripheral blood mononuclear cells of patients with thyroid dysfunction. Cytometry B Clin Cytom 2006; 70: 20-23.

17. Campo G, Ferraresi P, Marchesini J, et al. Relationship between paraoxonase Q192R gene polymorphism and on-clopidogrel platelet reactivity over time in patients treated with percutaneous coronary intervention. J Thromb Haemost 2011; 9: 2106-2108.

18. Campo G, Parrinello G, Ferraresi P, et al. Prospective evaluation of on-clopidogrel platelet reactivity over time in patients treated with percutaneous coronary intervention relationship with gene polymorphisms and clinical outcome. J Am Coll Cardiol 2011; 57: 2474-2483.

19. Brar SS, ten Berg J, Marcucci R, et al. Impact of platelet reactivity on clinical outcomes after percutaneous coronary intervention. A collaborative meta-analysis of individual participant data. J Am Coll Cardiol 2011; 58: 1945-1954. 
20. Mehran R, Rao SV, Bhatt DL, et al. Standardized bleeding definitions for cardiovascular clinical trials: a consensus report from the Bleeding Academic Research Consortium. Circulation 2011; 123: 2736-2747.

21. Cutlip DE, Windecker S, Mehran R, et al. Clinical end points in coronary stent trials: a case for standardized definitions. Circulation 2007; 115: 2344-2351.

22. Andell P, James SK, Cannon CP, et al. Ticagrelor Versus Clopidogrel in Patients With Acute Coronary Syndromes and Chronic Obstructive Pulmonary Disease: An Analysis From the Platelet Inhibition and Patient Outcomes (PLATO) Trial. J Am Heart Assoc 2015; 4: e002490.

23. Wakabayashi K, Gonzalez MA, Delhaye C, et al. Impact of chronic obstructive pulmonary disease on acute-phase outcome of myocardial infarction. Am J Cardiol 2010; 106: 305-309.

24. Berger JS, Sanborn TA, Sherman W, Brown DL. Effect of chronic obstructive pulmonary disease on survival of patients with coronary heart disease having percutaneous coronary intervention. Am J Cardiol 2004; 94: 649-651.

25. Wallentin L, Becker RC, Budaj A, et al. Ticagrelor versus clopidogrel in patients with acute coronary syndromes. N Engl J Med 2009; 361: 1045-1057.

26. Bonello L, Laine M, Kipson N, et al. Ticagrelor increases adenosine plasma concentration in patients with an acute coronary syndrome. J Am Coll Cardiol 2014; 63: 872-877.
27. Warnholtz A, Ostad MA, Velich N, et al. A single loading dose of clopidogrel causes dose-dependent improvement of endothelial dysfunction in patients with stable coronary artery disease: results of a double-blind, randomized study. Atherosclerosis 2008; 196: 689-695.

28. Mangiacapra F, Panaioli E, Colaiori I, et al. Clopidogrel versus ticagrelor for antiplatelet maintenance in diabetic patients treated with percutaneous coronary intervention: results of the CLOTILDIA Study. Circulation 2016; 134 835-837.

29. Ceconi C, Fox KM, Remme WJ, et al. ACE inhibition with perindopril and endothelial function. Results of a substudy of the EUROPA study: PERTINENT. Cardiovasc Res 2007; 73: 237-246.

30. Boodhwani M, Nakai Y, Voisine P, et al. High-dose atorvastatin improves hypercholesterolemic coronary endothelial dysfunction without improving the angiogenic response. Circulation 2006; 114: I402-408.

31. Husted S, Storey RF, Harrington RA, et al. Changes in inflammatory biomarkers in patients treated with ticagrelor or clopidogrel. Clin Cardiol 2010; 33: 206-212.

32. Campo G, Pavasini R, Pollina A, et al. On-treatment platelet reactivity in patients with chronic obstructive pulmonary disease undergoing percutaneous coronary intervention. Thorax 2014; 69: 80-81. 